\title{
Water-soluble BODIPY-nido-carborane nanoparticles applied to biocompatibility tumor cell imaging
}

\author{
Dongfang Dai ${ }^{1} \cdot$ Guangchang Lian $^{2} \cdot \mathrm{Xia} \mathrm{He}^{1} \cdot$ Jifeng Feng ${ }^{1} \cdot$ Guofan $\mathrm{Jin}^{2}$
}

Received: 29 September 2021 / Accepted: 24 November 2021 / Published online: 21 January 2022

(c) The Author(s), under exclusive licence to European Photochemistry Association, European Society for Photobiology 2022

\begin{abstract}
In this article, $o$-carborane has a high boron content, high hydrophobicity, and good chemical stability. It has been widely used in the fields of biology and medicine, especially in the application of boron neutron capture therapy (BNCT). However, $o$-carborane is a fat-soluble compound, its hydrophobicity is too strong, and its bioavailability is poor. This project aims to improve the water solubility of $o$-carborane drugs, so that the drugs can reach specific sites. For this reason, this article provides a one-pot reaction for the synthesis of water-soluble boron-containing drugs. 2-Chloro-1-(difluoroboranyl)5-((4-ethyl-3,5-dimethyl-2H-pyrrol-2-ylidene)(phenyl) methyl)-1H-pyrrole and ethylenediamine are used as raw materials to synthesize fluorescent molecular probe BODIPY- $\mathrm{NH}_{2}$, and the fluorescent molecular probe is reacted with P-CBMA (poly(carboxybetaine methacrylate)) to produce a water-soluble gel polymer. Water-soluble $o$-carborane polymers were synthesized by hydrogen bonding of the polymers with bis(4-azaspiro[3.4]octan-4-ium)-nido-ortho-carborane and bis(5azaspiro[4.5]decan-5-ium)-nido-ortho-caborane. The two polymers were characterized and the results showed that the maximum UV absorption wavelength of the two boron polymers in different polar solutions was $530-540 \mathrm{~nm}$. In the fluorescence spectrum, the maximum emission wavelengths of the two boron polymers are concentrated between 550 and $560 \mathrm{~nm}$. Through electron microscopy imaging, the fluoroboron pyrrole polymers wrap the boron clusters to form a spherical stacked. Through fluorescent cell imaging, both boron polymers can enter target cells.
\end{abstract}

Keywords BNCT $\cdot$ Carborane $\cdot$ BODIPY $\cdot$ Fluorescence $\cdot$ Biocompatibility

\section{Introduction}

Xia He and Jifeng Feng mainly contributed to the selection and cell imaging of cancer cells and tumor cells in the paper. Guofan Jin mainly contributed to the design, synthesis and characterization of boranes in this paper.

Guofan Jin

organicboron@ujs.edu.cn

Xia He

hexiabm@163.com

Jifeng Feng

jifeng_feng@163.com

1 Department of Radiotherapy, The Affiliated Cancer Hospital of Nanjing Medical University, Jiangsu Cancer Hospital, Jiangsu Institute of Cancer Research, Nanjing, People's Republic of China

2 School of Pharmacy, Jiangsu University, Zhenjiang 212013, People's Republic of China
Boron neutron capture therapy is an emerging therapeutic approach aimed at improving treatment rates for traditionally refractory tumors [1-3]. Boron cluster compounds represent a unique class of boron-containing compounds, not only because of their special coordination and unique three-dimensional structure, but also because of their wide range of applications from ceramics to BNCT reagents [4, 5]. From a practical point of view, on one hand, the cluster structure increases the concentration of boron, making them excellent candidates for BNCT reagents [6,7]; On the other hand, this compact structure also improves chemical and thermal stability, which enables them to be used in aerospace coatings, among other applications. Therefore, boron cluster compounds have been widely used as building blocks for the construction of functional polymeric materials with adjustable structure, amphiphilicity, and biocompatibility. With the development of polymerization technology, many 
polymers containing borane clusters have been developed in the past 2 decades.

Boron-containing compounds now have applications in many fields, including pharmaceutical chemistry, and are the epitome of a new class of drugs used by pharmaceutical chemists in drug design [8]. Carboranes are a class of organometallic compounds containing carbon, boron, and hydrogen atoms. They are the most widely studied boron compounds in pharmaceutical chemistry. Boron clusters have the following properties that are useful in drug design: (a) their unique non-covalent interaction capabilities, including ion interaction, $\sigma$-hole bonding, and dihydrogen-bond formation; $[9,10](2)$ it is helpful to construct the ellipsoid or spherical geometric structure and three-dimensional arrangement of three-dimensional molecules; (c) different boron clusters used can lead to different hydrophilicity, which can alter biological stability and pharmacokinetics; (d) resistance to ionizing radiation, a characteristic that is crucial to the design of radiopharmaceuticals. The most widely studied boron-containing compounds in pharmaceutical chemistry are carboranes, a class of caged boranes with highly delocalized electron-hydrophobic surfaces that are considered to be either inorganic benzene or three-dimensional aromatic compounds. Drug delivery for cancer treatment is also a hot topic worthy of research, including boron neutron capture therapy, especially $\mathrm{Fe}_{3} \mathrm{O}_{4}$-based nanoparticles and their surface modification with therapeutic substances [11] have attracted the attention of many scholars. In addition to the application of $\mathrm{Fe}_{3} \mathrm{O}_{4}$, other metals or metal oxides have also been successfully used to prepare functional nanoparticles $[12,13]$, which provides great potential for the development of new pharmaceutical materials.

On the other hand, living cell fluorescence microscopy is one of the most powerful multifunctional imaging tools for studying molecular-level mechanisms. Fluorescent dyes played a major role, and the modification strategies were as follows: (1) cell permeability [14, 15]; (2) high and obvious fluorescence [16]; (3) biocompatibility [17]; (4) cytotoxicity; (5) specific recognition and intracellular localization. Therefore, fluorophore based on BODIPY has been widely developed and applied in photocatalysis and photodynamic therapy fluorescent indicator, since it was first reported in 1968 [18]. It is a promising small molecular dye with strong application and development prospect. Its structural modifications are often aimed at improving the photophysical properties and biologically related activities. It is well known that substitution reaction is the most common reaction between carborane and fluoroboron pyrrole, usually modifying the side chain of fluoroboron pyrrole and introducing some halogen or amino groups. In addition, metal catalyzed coupling reactions have been reported, and palladium is a common catalyst. Electrocatalysis, a new way without chemical oxidant or metal catalyst, also provides an idea for the preparation of new fluorescent carborane.

BNCT can be used as a promising treatment for malignancies, but before BNCT can be used as a useful cancer treatment, the inherent disadvantage of carboranes is their high lipophilicity, making compounds containing potentially bioactive carboranes insoluble in water [19-21]. The introduction of a hydrophilic component into the carborane structure can greatly improve water solubility and thus simplify drug administration in vivo [22-25]. To improve the drug solubility, make the drug reach the target site smoothly, and observe the drug distribution in the cell, we designed a simple, efficient fluorescent molecular probe to make the intracellular visualization $[26,27]$. At the same time, P-CBMA was used as a water-soluble intermediate carrier to improve water solubility and biocompatibility. At the same time, it overcomes the shortcoming of small molecule boron-containing drugs, and the synthesis process is simple, and it has strong potential as an effective boron treatment drug.

\section{Experimental}

\subsection{General materials}

Purchased commercially, all solvents and reagents in this paper were analytical grade and used directly without further purification. ${ }^{1} \mathrm{H}$ NMR spectra were recorded on Bruker Avance II spectrometer in $\mathrm{CDCl}_{3}\left(400 \mathrm{MHz}\right.$ for $\left.{ }^{1} \mathrm{H}\right)$ : the chemical shift is expressed in ppm, and tetramethylsilane (TMS) is used as the internal standard. The infrared of the samples was recorded by Nicolet avato-370 FT-IR analyzer and tested by $\mathrm{KBr}$ tablet.

\subsubsection{Preparation of BODIPY- $\mathrm{NH}_{2}(2)$}

(Z)-2-chloro-1-(difluoroboranyl)-5-((4-ethyl-3,5-dimethyl2H-pyrrol-2-ylidene)(phenyl)methyl)-1H-pyrrole [28] (BODIPY-Cl, $0.5 \mathrm{~g}, 1.4 \mathrm{mmol}$ ) was dissolved in acetonitrile $(15 \mathrm{~mL})$, and ethylenediamine $(300 \mu \mathrm{L}, 4.5 \mathrm{mmol})$ and triethylamine $(600 \mu \mathrm{L}, 4.3 \mathrm{mmol})$ were added. The reaction was stopped after magnetic stirring at room temperature for $6 \mathrm{~h}$. Compound 2 (BODIPY-NH $, 0.45 \mathrm{~g}, 85 \%$ ) was isolated by column chromatography (petroleum ether: EtOAc $=1: 1$; dichloromethane: $\mathrm{MeOH}=25: 1) .{ }^{1} \mathrm{H}$ NMR $\left(400 \mathrm{MHz}, \mathrm{CDCl}_{3}\right): \delta(\mathrm{ppm})=8.01(\mathrm{~s}, 1 \mathrm{H}), 7.42-7.39(\mathrm{~m}$, $3 \mathrm{H}), 7.31-7.29(\mathrm{~m}, 2 \mathrm{H}), 6.46(\mathrm{~d}, J=4.4 \mathrm{~Hz}, 1 \mathrm{H}), 5.92(\mathrm{~d}$, $J=4.8 \mathrm{~Hz}, 1 \mathrm{H}), 3.41(\mathrm{~s}, 2 \mathrm{H}), 3.00(\mathrm{t}, J=6.0 \mathrm{~Hz}, 2 \mathrm{H}), 2.47$ (s, 3H), 2.35-2.29 (dd, $J=7.6,14.8 \mathrm{~Hz}, 2 \mathrm{H}), 1.37$ (s, 3H), $1.26(\mathrm{~s}, 2 \mathrm{H}), 0.99$ (t, $J=7.6 \mathrm{~Hz}, 3 \mathrm{H}) .{ }^{13} \mathrm{C}$ NMR $(100 \mathrm{MHz}$, $\left.\mathrm{CDCl}_{3}\right): \delta(\mathrm{ppm})=159.8,144.9,135.2,133.3,133.1,132.4$, 132.3, 130.0, 129.6, 128.3, 128.0, 106.0, 46.8, 41.4, 29.7, $17.1,15.0,11.9,11.6$. 


\subsubsection{Preparation of polymer 4}

Compound $2(0.05 \mathrm{~g})$ was dissolved in dichloromethane $(4.0 \mathrm{~mL}), \mathrm{P}-\mathrm{CBMA}(0.11 \mathrm{~g})$ and $\mathrm{NaHCO}_{3}(0.1 \mathrm{~g})$ were added, and the reaction was complete after $24 \mathrm{~h}$ at room temperature. $0.1 \mathrm{~g}$ Potassium tert-butanol was added to finish the reaction after $2 \mathrm{~h}$, and the reaction solution was filtered. Deep red polymer $4(0.14 \mathrm{~g})$ was obtained.

\subsubsection{Preparation of polymers 7 and 8}

Polymer $4(0.07 \mathrm{~g})$ was dissolved in $3.0 \mathrm{~mL}$ tetrahydrofuran, and then, Bis(4-azoronium [3.4]octane-4-fonium) - $o$-carborane $(0.015 \mathrm{~g})$ was added. The reaction was carried out by magnetic stirring at room temperature for $12 \mathrm{~h}$, and the polymer 7 was obtained. 7: ${ }^{11} \mathrm{~B}$ NMR $(128 \mathrm{MHz}$, $\left.\mathrm{CDCl}_{3}\right): \delta(\mathrm{ppm}) 1.27(\mathrm{~s}),-1.00(\mathrm{~s}),-11.17(\mathrm{~s}),-17.20$ (s), -21.95 (s). 8: ${ }^{11} \mathrm{~B}$ NMR (128 MHz, $\left.\mathrm{CDCl}_{3}\right): \delta(\mathrm{ppm})$ 0.57 (d, $J=146.8 \mathrm{~Hz}),-11.26(\mathrm{~s}),-17.29(\mathrm{~s}),-22.01$ (s) (Table 1).

\subsection{Typical procedure for measuring the UV-Vis spectra}

UV-visible spectrophotometer UV-2550 was used to record the ultraviolet profile of the compound, using a quartz cuvette, and the absorption wavelength range was set at 200-800 $\mathrm{nm}$. The $3 \mathrm{mg}, 7 \mathrm{mg}$, and $8 \mathrm{mg}$ polymers were dissolved in $2 \mathrm{ml}$ and $4 \mathrm{ml} \mathrm{DCM}$, respectively, to produce two different sample concentrates. The concentrated solution with the volume of $20 \mu \mathrm{L}, 40 \mu \mathrm{L}, 60 \mu \mathrm{L}, 80 \mu \mathrm{L}$, and $100 \mu \mathrm{L}$ was dissolved in $3 \mathrm{~mL} \mathrm{DCM}$, and DCM was used as blank control. In addition, dichloromethane, methanol, ethanol, tetrahydrofuran, and ethyl acetate were used as solvents for testing.

\subsection{Typical procedure for collecting fluorescence spectra}

A Shimadzu RF-5301PCS fluorescence spectrophotometer was used to collect fluorescence profiles. The absorption wavelength was set at 300-800 $\mathrm{nm}$ and the excitation wavelength was set at $500 \mathrm{~nm}$. Similar to the UV test, two different sample concentrates were prepared by dissolving $3 \mathrm{mg}$, $7 \mathrm{mg}$, and $8 \mathrm{mg}$ of polymer in $2 \mathrm{ml}$ and $4 \mathrm{~mL}$ of DCM, respectively. Similarly, fluorescence spectra of $20 \mu \mathrm{L}, 40 \mu \mathrm{L}$, $60 \mu \mathrm{L}, 80 \mu \mathrm{L}$, and $100 \mu \mathrm{L}$ of the original solution dissolved in $3 \mathrm{~mL}$ of different solvents were collected.

\subsection{Living cell imaging}

Materials: HeLa cells in logarithmic growth phase. First, trypsinization was performed, and the cells were inoculated in a 96-well plate containing a circular cap, placed in a 5\% $\mathrm{CO} 2$ incubator, cultured at $37^{\circ} \mathrm{C}$ for $24 \mathrm{~h}$, and adhered to the wall. The stock solution $(20 \mathrm{mg} / \mathrm{mL})$ was prepared by dissolving polymers 7 and 8 in DMSO. Again, the solution was diluted to a suitable concentration using DMSO. The original culture medium of cells in each well was replaced with culture medium containing $10 \mu \mathrm{g} / \mathrm{mL}$ different samples. After treatment for $24 \mathrm{~h}$, the culture medium was discarded and washed with PBS twice, and paraformaldehyde fixative was added for $10 \mathrm{~min}$. The fixative was removed and rinsed with PBS for 2 times, and then, DAPI was incubated in the dark for 10 min. Finally, the staining solution was discarded and washed with PBS twice. After treatment with anti-fluorescence quenching scaffold, fluorescence images of cells were obtained under fluorescence microscope.

\subsection{Characterization}

Transmission electron microscope (TEM) maps were collected on the Zeiss Ultra Plus at an accelerated voltage of $15 \mathrm{keV}$ with the Oxford Instruments X-Max $60 \mathrm{~mm}^{2}$ SDD $\mathrm{X}$-ray microanalysis system attached. The sample was added to ethanol, the suspended precipitate was added to the silicon wafer, the sample was attached to the sample tray with conductive adhesive, and TEM imaging was performed by scanning electron microscope at $0.5 \mu \mathrm{m}$ and $200 \mathrm{~nm}$ scales.

\section{Results and discussion}

\subsection{Design and synthesis}

Overall experimental route: Therefore, first of all, N1-(1(difluoroboroyl-3, 5-dimethyl-2H-pyrrole-2-subunit) (phenyl) methyl)-1 was synthesized by nucleophilic substitution of 2-chloro-1-(difluoroboroyl-3, 5-dimethyl-2H-pyrrole2-subunit) (phenyl) methyl)-1H-pyrrole with ethylenediamine H-pyrrole-2-yl) ethane-1, 2-diamine (compound 2). Compound 2 was substituted with P-CBMA under alkaline condition to produce water-soluble fluorescent polymer. Bis (4-azoronium [3.4] octane-4-fonium)- $o$-carborane and Bis (5-azoronium [4.5] decane-5-fonium)-o-carborane [29] were coated in water-soluble fluorophore to obtain the products. The reaction route is as follows (Scheme 1).

\subsection{Photophysical properties}

To preliminarily determine the structural characteristics of the two carborane polymers, the characteristic peaks of the main functional groups were analyzed by hydrogen NMR spectroscopy. As shown in the hydrogen spectra in Fig. 3-1, the three compounds all had multiple aromatic characteristic peaks between 6.0 and 8 ppm. Polymers 7 and 8 can 
Table 1 Synthesis and side chain substituents of fluoroboropyrrole derivatives

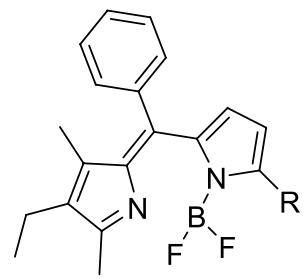

\begin{tabular}{|c|c|c|}
\hline Compound & $\mathrm{R}$ & Structure \\
\hline 1 & \} & \\
\hline 2 & & \\
\hline 3 & - & \\
\hline
\end{tabular}

4<smiles>CNCCN(CC(C)(C)C(=O)OCC[N+](C)(C)CCC(=O)[O-])CC(C)(C)C(=O)OCC[N+](C)(C)CCC(=O)[O-]</smiles>

5

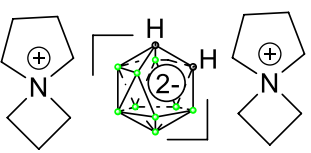

6

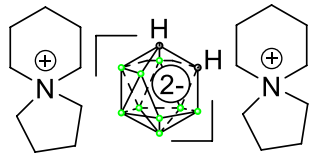

7

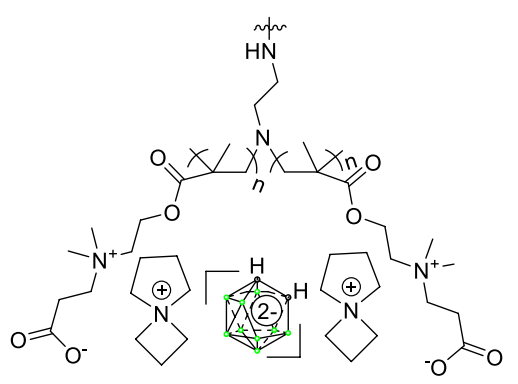

8

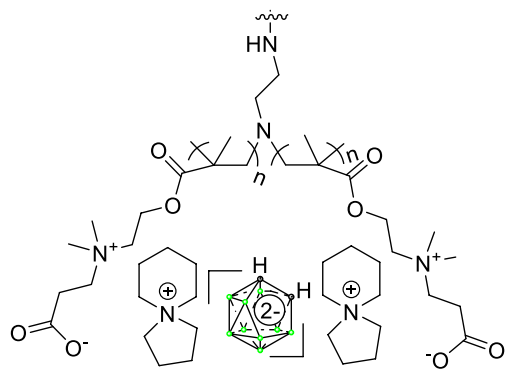



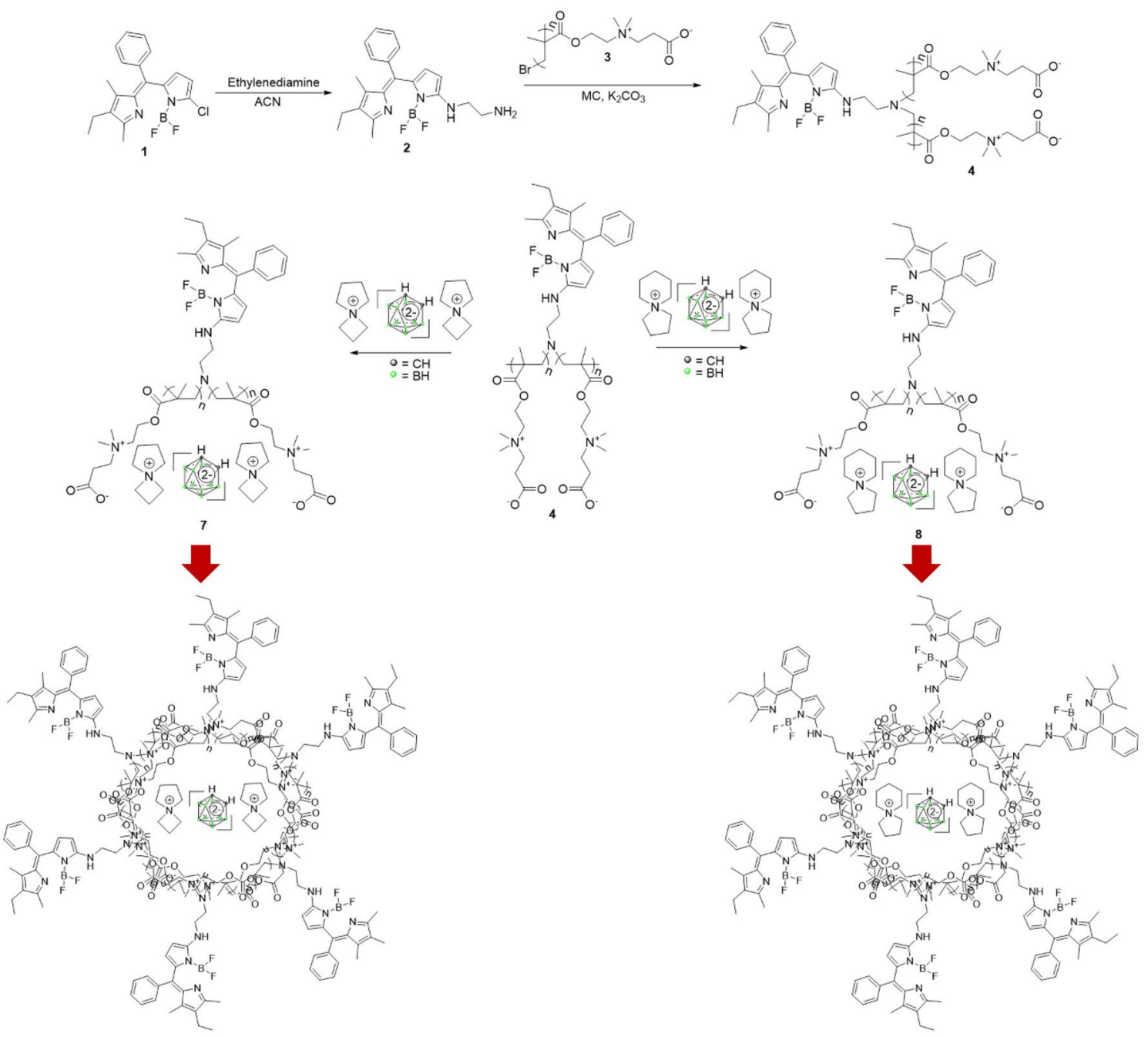

Scheme 1 General synthetic routes

Table 2 Photophysical of polymers 7 and 8 in different solvents

\begin{tabular}{lll}
\hline Polymers & $\mathbf{7}$ & $\mathbf{8}$ \\
\hline$\lambda_{\text {abs max }}, \mathrm{nm}$ & & \\
DCM & 541 & 540 \\
$\mathrm{MeOH}$ & 543 & 542 \\
$\mathrm{EtOH}$ & 542 & 538 \\
THF & 544 & 539 \\
EA & 546 & 542 \\
$\lambda_{\text {em max }}, \mathrm{nm}$ & & \\
DCM & 553 & 552 \\
MeOH & 550 & 550 \\
EtOH & 551 & 550 \\
THF & 554 & 551 \\
EA & 551 & 551 \\
\hline
\end{tabular}

be observed to have obvious convex peaks between 0.5 and $3.0 \mathrm{ppm}$ in the high magnetic field, and irregular cluster peaks can be seen when superimposed with the branched polymer peaks, which can be regarded as BH groups (Figs. S2 and S3). In addition, a spike was observed at $1.27 \mathrm{ppm}$ and $0.57 \mathrm{ppm}$ in the boron NMR spectroscopy of compounds $\mathbf{7}$ and $\mathbf{8}$, respectively, which is the chemical shift of the common boron tricoordination compounds (see supporting information).

Infrared spectroscopy can be used to study the structure and chemical bonds of molecules, the position of infrared absorption peak, and the strength of the reaction of molecular structure characteristics. The characteristic absorption peaks of multiple aromatic groups in the vicinity of $3000 \mathrm{~cm}^{-1}$ and $1500 \mathrm{~cm}^{-1}$ of the two carborane polymers were observed by infrared spectroscopy. The characteristic peak of $\mathrm{BH}$ is usually in the range of $2560-2600 \mathrm{~cm}^{-1}$. However, due to the open basket-like structure of the boron cluster of the 
two boron-containing polymers, the absence of single boron atom in the structure leads to the blue shift of absorption peak, so the absorption peak near $2500-2600 \mathrm{~cm}^{-1}$ can be considered as boron spectrum absorption of carborane (Fig. S4) (see supporting information).

To better observe the photophysical properties of the two polymers in solution, different polar solvents such as dichloromethane, methanol, ethanol, tetrahydrofuran, and ethyl acetate were selected as solvent media for spectral analysis. Among the five solvents, the ultraviolet absorption spectra of both carborane polymers are similar. The maximum absorption wavelengths of the two polymers are in the range of 530-540 $\mathrm{nm}$ (Table 2). In general, the absorption spectra are mainly concentrated in the structure of the
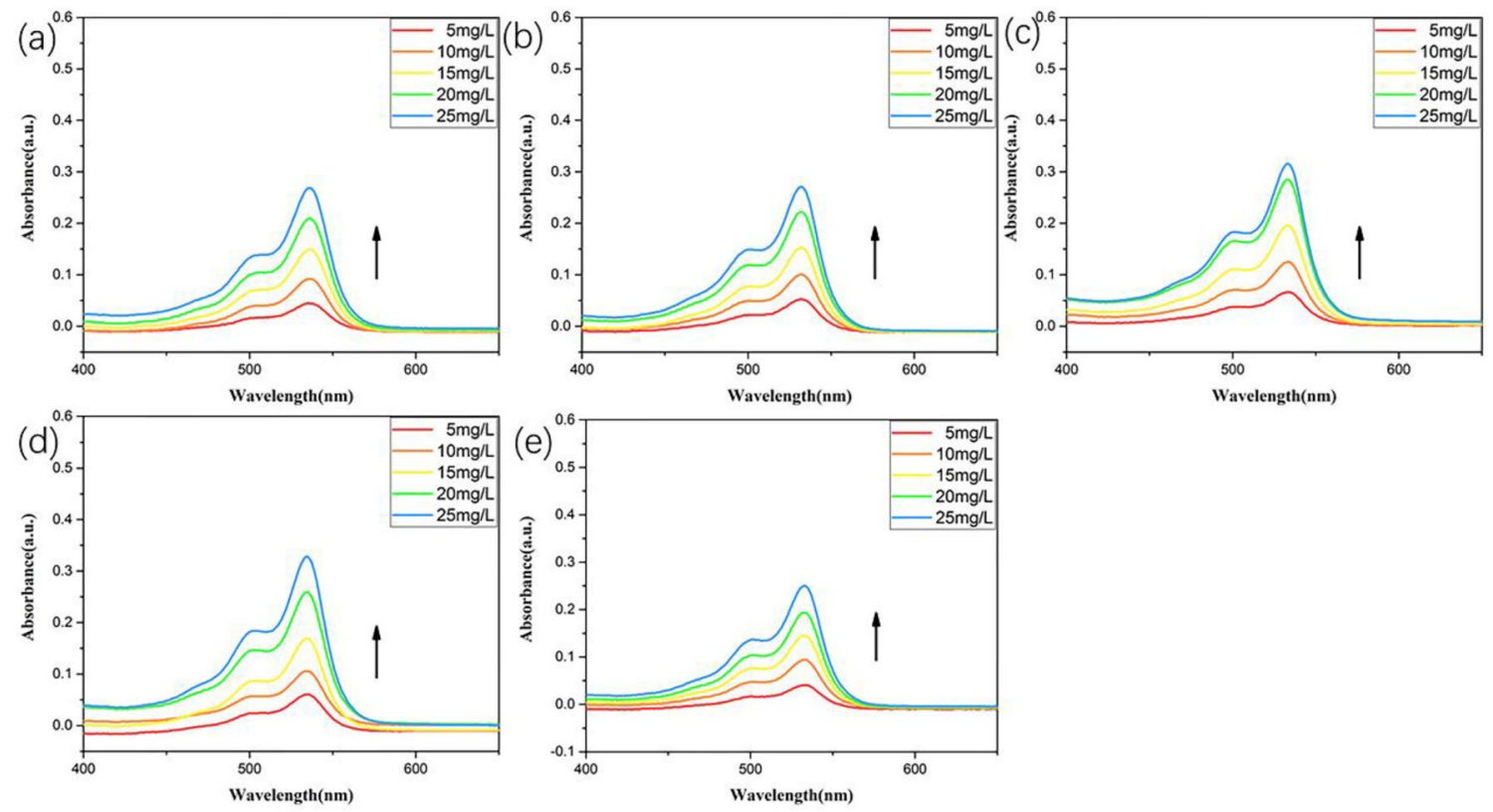

Fig. 1 UV absorption spectrum: a DCM; b MeOH; c EtOH; d THF; e EA of polymer 7
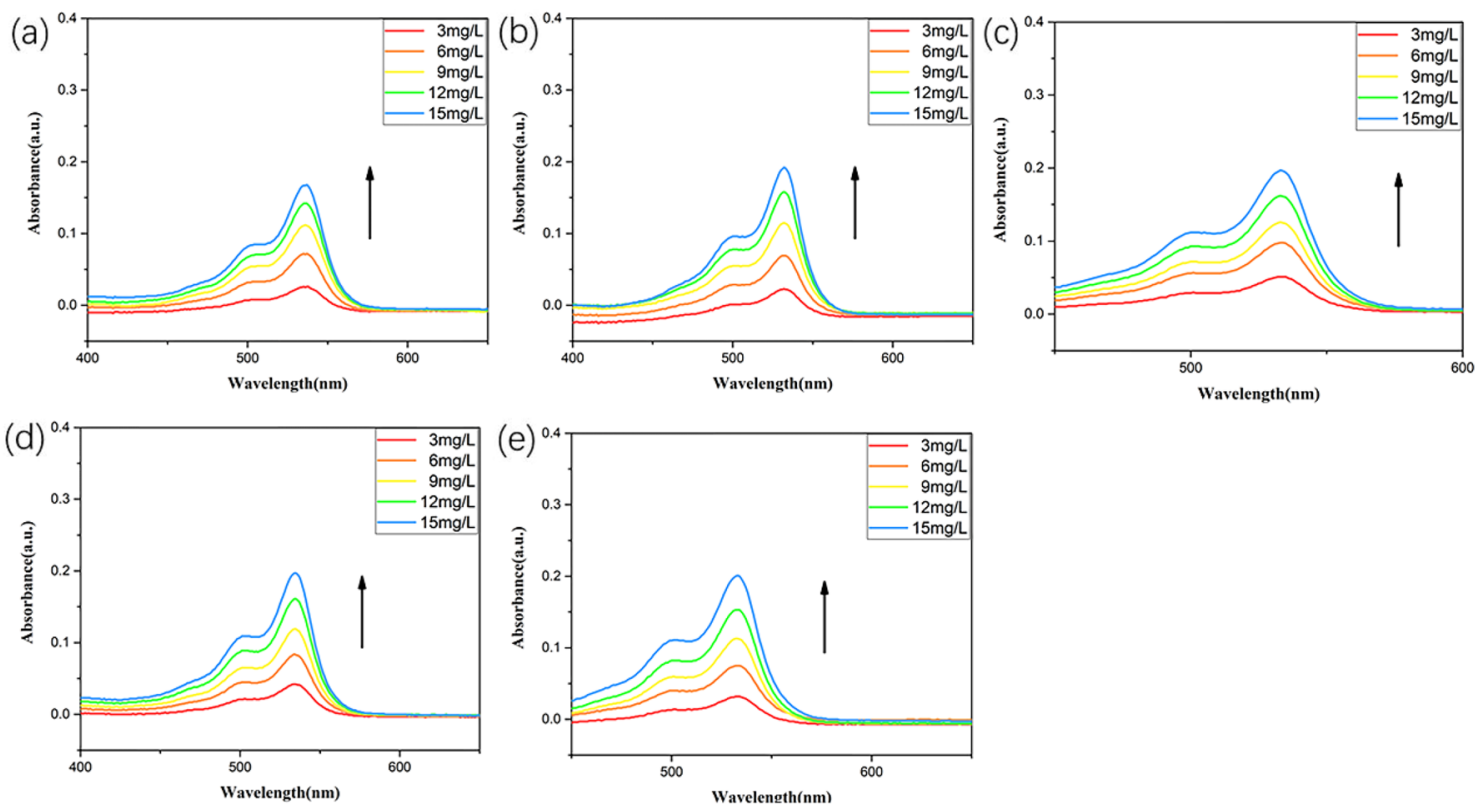

Fig. 2 UV absorption spectrum: a DCM; b MeOH; c EtOH; d THF; e EA of polymer 8 
FBDI-pyrrole polymer, and the branched functional groups have no significant effect. With the increase of concentration, the absorbance of the two boron polymers increased significantly (Figs. 1 and 2).

Similarly, the fluorescence emission spectra of the two polymers in five different solvents, such as dichloromethane, methanol, ethanol, tetrahydrofuran, and ethyl acetate, were measured. The fluorescence emission spectra of the two carborane polymers in five different solvents were similar, and the wavelengths were concentrated in the range of 550-560 $\mathrm{nm}$ (Table 2). The fluorescence intensity of the two carborane polymers increased with increasing concentrations (Figs. 3 and 4).

From micro level to better analyze the concrete forms of two kinds of polymer, visually observed two carbon borane polymer morphology, by transmission electron microscopy (tem) imaging, as shown in Figs. 3, 4, 5, 6, 7, can observe polymers $\mathbf{7}$ and $\mathbf{8}$ internal spherical together and polymers 8 nanoparticles than $\mathbf{7}$ more closely. This is because the amphoteric ions at the end of the fluoro-boro-pyrrole fluorescent polymer are closely bound with the o-carborane derivatives through ionic bonds, forming small particle nanoclusters that gather together (Fig. 5).

\subsection{Cell imaging}

To observe the selectivity, biocompatibility, and permeability of two carborane polymers in target cells, cell imaging studies were conducted. The imaging of two boroncontaining polymers in cancer cells was tested. Through the cell imaging of two boron-containing polymers, as shown in Figs. 3, 5, 6, it can be clearly seen that both boron-containing polymers can penetrate the cell membrane into the cytoplasm and penetrate the nucleus, and both polymers have high selectivity and high biocompatibility (Fig. 6).

\section{Conclusion}

As a promising cancer treatment, BNCT still has many problems, and further research and development of boron polymers are needed to improve the treatment rate and reduce the potential toxicity. In this paper, two boron-containing polymers were synthesized to solve the problems of poor water solubility of current drugs used in BNCT and low drug content in cancer cells. From the optical properties of the two compounds, the Stokes shift is small, and the effect of branched functional groups on the absorption spectrum is not significant. Fluoro-boron-pyrrole chloride was used as the fluorophore core, and the side chain was linked to ethylenediamine to enhance the fluorescence intensity, so that the selectivity of drugs and drug content in cells could be monitored quickly, simply, and conveniently. To solve the insoluble property of carborane in water, the water-soluble carrier P-CBMA was introduced to increase the hydrophilicity of the structure, and thus the preparation of excellent biocompatibility, hydrophilic drugs. It provides a new idea for the research and design of BNCT. These results have (a)

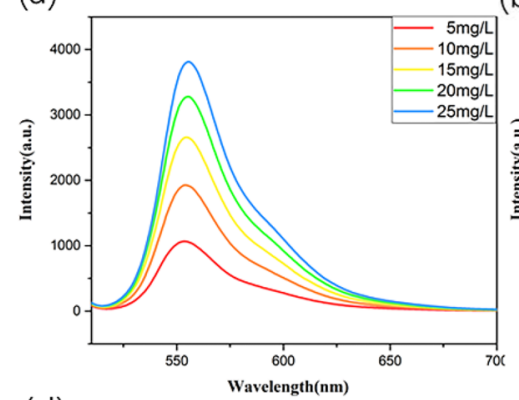

(d)

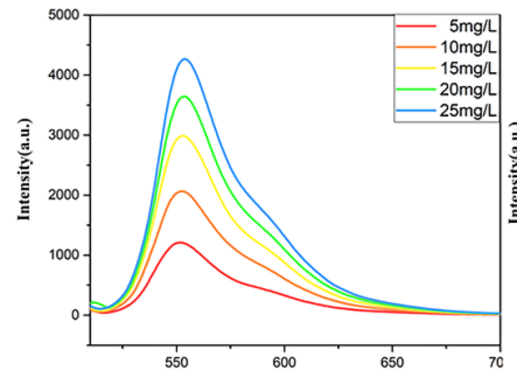

(b)

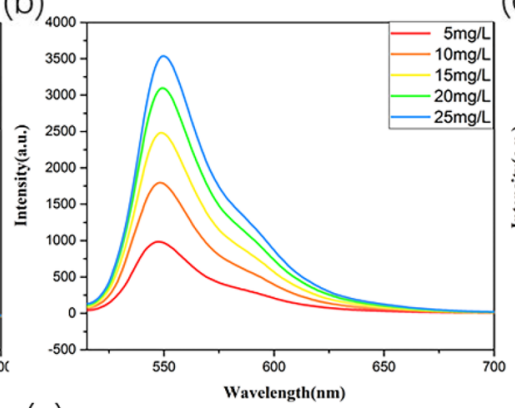

(e)

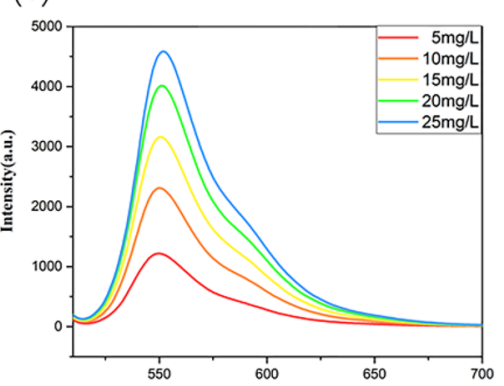

(c)

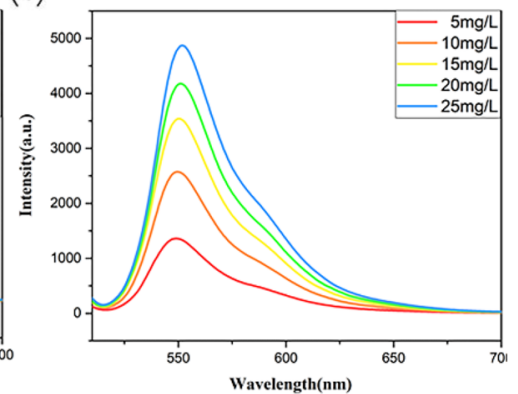

Fig. 3 Fluorescence spectrum: a DCM; b MeOH; c EtOH; d THF; e EA of polymer 7 

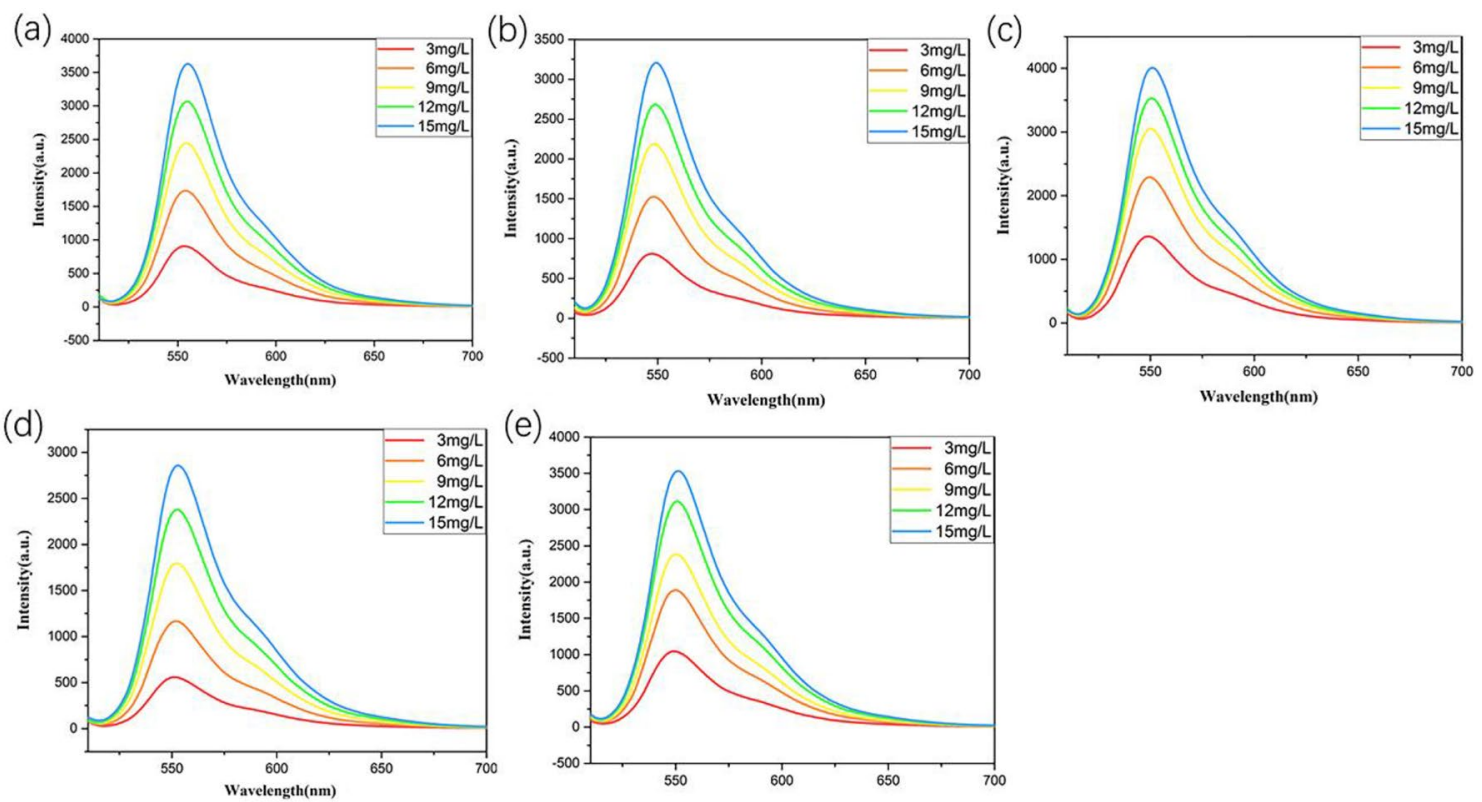

Fig. 4 Fluorescence spectrum: a DCM; b MeOH; c EtOH; d THF; e EA of polymer 8

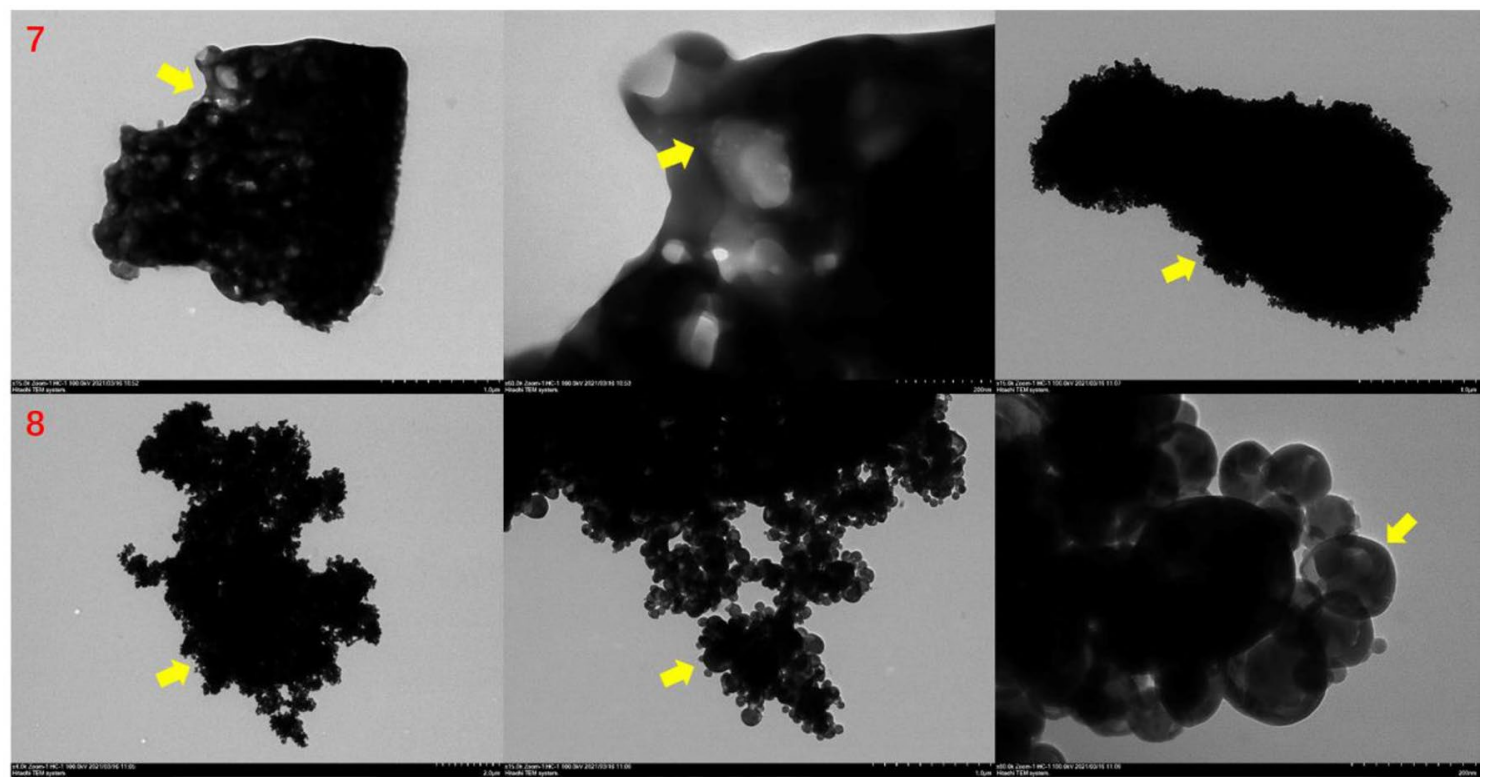

Fig. 5 TEM imaging of polymers 7 and 8

important implications for the use of carborane polymers as BNCT agents for the treatment of cancer. In the future research, the research on the optical properties of polymers and the antitumor activity of polymers by branched chains can be further explored to pursue carborane drugs with better hydrophilicity, strong cytotoxicity to tumor cells, and low toxicity to normal cells. 


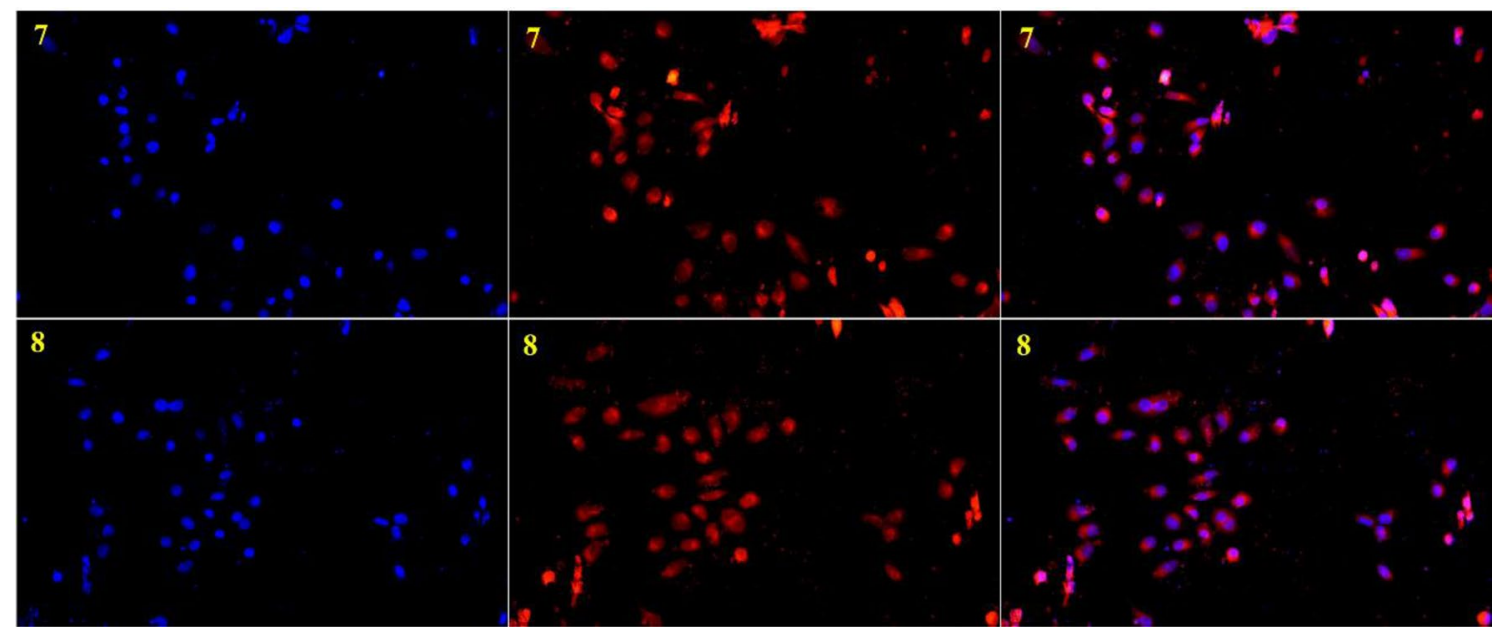

Fig. 6 Fluorescence imaging of polymer 7 and 8

Supplementary Information The online version contains supplementary material available at https://doi.org/10.1007/s43630-021-00148-1.

Acknowledgements This research was supported by the grants from Jiangsu Health Commission (M2021021), China Postdoctoral Science Foundation (2021M701499), Jiangsu postdoctoral Foundation (2021Z282), the Scientific Research Foundation of Jiangsu University (Grant No. 17JDG002).

\section{Declarations}

Conflict of interest The authors declare that they have no known competing financial interests or personal relationships that could have appeared to influence the work reported in this paper.

\section{References}

1. Lin, K. H., Chen, Y. W., Lee, C. R., \& Huang, W. S. (2019). Nuclear theranostics in Taiwan. Nuclear Medicine and Molecular Imaging, 53, 86-91. https://doi.org/10.1007/s13139-019-00576-Z

2. Naresh, K., Raviraj, V., \& Hwang, K. C. (2017). Engineering novel targeted boron-10-enriched theranostic nanomedicine to combat against murine brain tumors via $\mathrm{mr}$ imaging-guided boron neutron capture therapy. Advanced Materials. https:// doi.org/10.1002/adma.201700850

3. Tetsuya, Y., \& Koji, T. (2009). Particle radiotherapy for malignant gliomas. Brain and Nerve, 61, 855-866.

4. Malouff, T. D., Seneviratne, D. S., Stross, W. C., Waddle, M. R., \& Ebner, D. K. (2021). Boron neutron capture therapy: A review of clinical applications. Frontiers in Oncology, 11, 601820. https://doi.org/10.3389/fonc.2021.601820

5. Barth, R. F., Soloway, A. H., Goodman, J. H., \& Tjarks, W. (1999). Boron neutron capture therapy of brain tumors: An emerging therapeutic modality. Neurosurgery, 44(433-50), 450-451. https://doi.org/10.1097/00006123-199903000-00001

6. Hawthorne, M. F., \& Lee, M. W. (2003). A critical assessment of boron target compounds for boron neutron capture therapy. Journal of Neuro-oncology, 62, 33-45. https://doi.org/10.1007/ BF02699932
7. Soloway, A. H., Hatanaka, H., \& Davis, M. A. (1967). Penetration of brain and brain tumor. VII. Tumor-binding sulfhydryl boron compounds. Journal of Medicinal Chemistry, 10, 714-717. https://doi.org/10.1021/jm00316a042

8. Olsson, P., Gedda, L., Goike, H., \& Jorgen, C. (1998). Uptake of a boronated epidermal growth factor-dextran conjugate in $\mathrm{CHO}$ xenografts with and without human EGF-receptor expression. Anti-Cancer Drug Design, 13, 279-289.

9. Lo, R., Fanfrlik, J., Lepsik, M., \& Hobza, P. (2015). The properties of substituted 3D-aromatic neutral carboranes: The potential for $\sigma$-hole bonding. Physical Chemistry Chemical Physics, 17, 20814-20821. https://doi.org/10.1039/C5CP03617H

10. Poater, J., Sola, M., Vinas, C., \& Teixidor, F. (2014). $\pi$ aromaticity and three-dimensional aromaticity: Two sides of the same coin. Angewandte Chemie, 53, 12191-12195. https://doi.org/10.1002/ anie.201407359

11. Tishkevich, D. I., Korolkov, I. V., Kozlovskiy, A. L., Anisovich, M., Vinnik, D. A., Ermekova, A. E., Vorobjova, A. I., Shumskaya, E. E., Zubar, T. I., Trukhanov, S. V., Zdorovets, M. V., \& Trukhanov, A. V. (2019). Immobilization of boron-rich compound on fe3o4 nanoparticles: Stability and cytotoxicity. Journal of Alloys and Compounds, 797, 573-581. https://doi.org/10.1016/j.jallcom. 2019.05.075

12. Vorobjova, A., Tishkevich, D., Shimanovich, D., Zdorovets, M., Kozlovskiy, A., Zubar, T., Vinnik, D., Dong, M., Trukhanov, S., Trukhanov, A., \& Fedosyuk, V. (2020). Electrochemical behaviour of ti/al2o3/ni nanocomposite material in artificial physiological solution: Prospects for biomedical application. Nanomaterials, 10, 173. https://doi.org/10.3390/nano10010173

13. Almessiere, M. A., Trukhanov, A. V., Khan, F. A., Slimani, Y., Tashkandi, N., Turchenko, V. A., Zubar, T. I., Tishkevich, D. I., Trukhanov, S. V., Panina, L. V., \& Baykal, A. (2020). Correlation between microstructure parameters and anti-cancer activity of the mn0.5zn0.5 (euxndxfe2-2x)o-4 nanoferrites produced by modified sol-gel and ultrasonic methods. Ceramics International, 46, 7346-7354. https://doi.org/10.1016/j.ceramint.2019.11.230

14. Xiao, F., Wang, Y., Shao, T., \& Jin, G. F. (2020). Acetonitrilated Unsymmetric BODIPYs having glycine fluorescence responsive quenching: Design, synthesis and spectroscopic properties. Spectrochimica Acta Part A, 233, 118211. https://doi.org/10.1016/j. saa.2020.118211 
15. Tang, X., Zhu, Z., Wang, Z. K., Tang, Y., Wang, L., \& Liu, L. (2020). Developed a novel quinazolinone based turn-on fluorescence probe for highly selective monitoring hypochlorite and its bioimaging applications. Spectrochimica Acta Part a-Molecular and Biomolecular Spectroscopy, 228, 117845. https://doi.org/10. 1016/j.saa.2019.117845

16. Li, W. J., Liu, D., Bi, X. Y., \& You, T. Y. (2020). Enzyme-triggered inner filter effect on the fluorescence of gold nanoclusters for ratiometric detection of mercury(ii) ions via a dual-signal responsive logic. Sensors and Actuators A, 302, 111794. https:// doi.org/10.1016/j.sna.2019.111794

17. Shao, T., Wang, Y., Xiao, F., \& Jin, G. F. (2021). Strategies of porous network quinolone polymers: A comprehensive evaluation of their biological activity. Dyes and Pigments, 184, 108809. https://doi.org/10.1016/j.dyepig.2020.108809

18. Zou, X. B., Jiang, C. P., Li, Z. H., Sun, Y., Shi, J. Y., Li, Y. X., Huang, X. W., Zhang, D., Hu, X. T., Zhai, X. D., \& Wei, X. O. (2020). A "turn on-off" fluorescent probe based on aptamer/ gold nanoparticles for rapid detection of isocarbophos. Chinese Journal of Analytical Chemistry, 48, 507-515. https://doi.org/10. 19756/j.issn.0253-3820.191335

19. Armstrong, A. F., \& Valliant, J. F. (2007). The bioinorganic and medicinal chemistry of carboranes: From new drug discovery to molecular imaging and therapy. Dalton Transactions, 38, 42404251. https://doi.org/10.1039/b709843j

20. Varadarajan, A., Hawthorne, M., \& Frederick, L. (1991). Novel carboranyl amino acids and peptides: Reagents for antibody modification and subsequent neutron-capture studies. Bioconjugate Chemistry, 2, 242-253. https://doi.org/10.1021/bc00010a008

21. Gianpiero, C., Anis, D., Eugen, B., \& John, T. (2018). Towards carborane-functionalised structures for the treatment of brain cancer. Drug Discovery Today, 23, 63-75. https://doi.org/10.1016/j. drudis.2017.08.009

22. Andrade, L. H., Barcellos, T., \& Santiago, C. G. (2010). Chemoenzymatic synthesis of boron-containing chiral amines and amides. Tetrahedron, 21, 2419-2424. https://doi.org/10.1016/j. tetasy.2010.09.011

23. Ebdrup, S., Jacobsen, P., \& Vedso, P. (2005). Structure-activity relationship for aryl and heteroaryl boronic acid inhibitors of hormone-sensitive lipase. Bioorganic and Medicinal Chemistry, 13, 2305-2312. https://doi.org/10.1016/j.bmc.2004.12.042

24. Lee, C. H., Jin, G. F., Ban, H. S., \& Nakamura, A. (2009). HNew types of potential BNCT agents, o-carboranyl aminoalcohols. Tetrahedron Letters, 50, 2960-2963. https://doi.org/10.1016/j.tetlet. 2009.03.207

25. Lee, C. H., Jin, G. F., \& Lee, J. D. (2007). Synthesis and characterization of polar functional group substituted mono- and bis-(o -carboranyl)-1,3,5-triazine derivatives. Tetrahedron Letters, 49, 159-164. https://doi.org/10.1016/j.tetlet.2007.10.145

26. Tang, Y., Li, Y. Y., Liu, L., Ni, H. C., Han, J., Wang, L., Mao, Y. L., Ni, L., \& Wang, Y. (2020). A water-soluble colorimetric and fluorescent probe for rapidly sensing of clo- in organisms. Journal of Photochemistry and Photobiology A, 387, 112166. https://doi. org/10.1016/j.jphotochem.2019.112166

27. Wang, Y., Xiao, F., Shao, T., Hu, K., \& Jin, G. F. (2021). A multiple acetal chalcone-BODIPY-based fluorescence: Synthesis, physical property, and biological studies. Analytical and Bioanalytical Chemistry, 413, 2529-2541. https://doi.org/10.1007/ s00216-021-03208-8

28. Keefe, K. A., Chahine, E. B., DiSaia, P. J., \& Berns, M. W. (2001). Fluorescence detection of cervical intraepithelial neoplasia for photodynamic therapy with the topical agents 5-aminolevulinic acid and benzoporphyrin-derivative monoacid ring. American Journal of Obstetrics and Gynecology, 184, 1164-1169. https:// doi.org/10.1067/mob.2001.113123

29. Lv, Z., Wang, Z., Xiao, F., \& Jin, G. F. (2020). Well defined rich electronic structure: facile approach for nido-carborane fused azaspirodecaniums in water solution. Journal of the Brazilian Chemical Society, 1, 170-174. https://doi.org/10.21577/01035053.20190152 